\title{
Laboratory formation of large molecules in the gas phase
}

\author{
Junfeng Zhen ${ }^{1,2}$ \\ ${ }^{1}$ CAS Key Laboratory for Research in Galaxies and Cosmology, Department of Astronomy, University of Science and Technology \\ of China, Hefei 230026, PR China \\ ${ }^{2}$ School of Astronomy and Space Science, University of Science and Technology of China, Hefei 230026, PR China \\ e-mail: jfzhen@ustc.edu.cn
}

Received 13 December 2018 / Accepted 25 January 2019

\begin{abstract}
We report the experimental study on the formation process of large molecules (e.g. a family group of molecular clusters and graphene) in the gas phase. The experiment was carried out using a quadrupole ion trap in combination with time-of-flight mass spectrometry. As the initial molecular precursor, dicoronylene (DC, $\left.\mathrm{C}_{48} \mathrm{H}_{20}\right)$ /anthracene $\left(\mathrm{C}_{14} \mathrm{H}_{10}\right)$ cluster cations, the results show that large PAH cluster cations (e.g., $\left(\mathrm{C}_{14} \mathrm{H}_{10}\right) \mathrm{C}_{48} \mathrm{H}_{n}^{+}, n=[1-19]$ and $\left.\left(\mathrm{C}_{14} \mathrm{H}_{10}\right) \mathrm{C}_{62} \mathrm{H}_{m}^{+}, m=[1-25]\right)$ and PAH-graphene cluster cations (e.g., $\left(\mathrm{C}_{14} \mathrm{H}_{10}\right)_{n} \mathrm{C}_{48}^{+}, n=0$, $1,2,3$ and $\left.\left(\mathrm{C}_{14} \mathrm{H}_{10}\right)_{m} \mathrm{C}_{62}^{+}, m=0,1,2\right)$ are formed by gas-phase condensation under laser irradiation conditions. We infer that these results present in here provide a formation route for interstellar large molecules under the influence of a strong radiation field in the ISM. The relevance of newly formed species to the nanometer-sized dust grain in space is briefly discussed.
\end{abstract}

Key words. astrochemistry - molecular processes - methods: laboratory: molecular - ultraviolet: ISM - evolution

\section{Introduction}

Mid-infrared (IR) spectra of the interstellar medium (ISM) are dominated by broad features at 3.3, 6.2, 7.7, 8.6 and $11.2 \mu \mathrm{m}$, these features are generally attributed to IR fluorescence of large ( 50-100 C-atom) polycyclic aromatic hydrocarbon (PAH) molecules pumped by ultraviolet (UV) photons (Sellgren 1984; Puget \& Leger 1989; Allamandola et al. 1989; Croiset et al. 2016). Interstellar IR spectra also show evidence of the presence of PAH clusters and very small dust grains (Van Diedenhoven et al. 2004; Rapacioli et al. 2005; Berné et al. 2007; Pilleri et al. 2015). In the ISM, PAH clusters or PAH related complex species are believed to be the self-assembled intermediaries between free gas-phase PAHs and amorphous carbon particles, PAH radicals formed by photo-dehydrogenation are discussed to be the driving force for the cluster formation (Henning \& Salama 1998; Richter et al. 2004; Rapacioli et al. 2006; Rhee et al. 2007).

Photo-dissociation regions (PDRs) provide a natural laboratory for the study of the interaction of UV photons with carbonaceous species (Pety et al. 2005; Rapacioli et al. 2005; Hollenbach \& Tielens 1999; Micelotta et al. 2010). Observations with the Spitzer Space Telescope and the Herschel Space Observatory of the prototypical PDR, NGC 7023, have revealed the important role of photochemistry in the destruction of interstellar PAHs (Malloci et al. 2008; Berné \& Tielens 2012). The top-down processes behind $\mathrm{PAH}$ breakdown initiated by UV irradiation or energetic particle bombardment are balanced by bottom-up molecular growth processes (Tielens 2013; Zhen et al. 2018a), where inside the benign environments of molecular clouds, small PAHs can collide and cluster into larger structures (Tielens 2008; Candian et al. 2018). So far, experimental evidence of this process has been lacking. Specifically, laboratory studies of processing of van der Waals clusters of PAHs and fullerenes by energetic ions (e.g., $24 \mathrm{keV} \mathrm{O}^{2+}$ or $12 \mathrm{keV} \mathrm{Ar}^{2+}$ ) have revealed the formation of chemically bonded large species through direct knock-out of carbon atoms (Zettergren et al. 2010, 2013; Chen et al. 2015; Delaunay et al. 2015).

In the present study, we obtained the formation process of large molecules $(\sim 100$ atoms, e.g., PAH clusters or graphene) under laser irradiation $(355 \mathrm{~nm}, 3.49 \mathrm{eV})$, the main aim is to investigate the inherent of large PAH cluster stabilities, the related fragmentation processes, and their collision reaction process (gas-phase condensation) at same time. We explored dicoronylene (DC, $\left.\mathrm{C}_{48} \mathrm{H}_{20}\right) /$ anthracene $\left(\mathrm{C}_{14} \mathrm{H}_{10}\right)$ cluster cations as the initial precursor, and experiments were performed by using of newly constructed set up: a quadrupole mass filterquadrupole ion trap source, analyzed by TOF mass spectrometry. We selected DC as it is an all-benzenoid PAH with size (48 C-atoms) in the astrophysically relevant range (Tielens 2008; Croiset et al. 2016) that possibly serves as a prototypical example for large(r) PAHs. The photo-fragmentation behavior and the IR spectra of $\mathrm{DC}^{+}$have been obtained (Zhen et al. 2018b; Castellanos et al. 2018), its IR spectra provides a better agreement with astronomical observations (Ricca et al. 2012).

\section{Experimental methods}

The experiments and the information on experimental procedures are available in Zhen et al. (2018c). We provide only the essential relevant details here. DC is evaporated by heating the powder (Kentax, with a purity better than $99.5 \%$ ) in the oven at a temperature of $\sim 633 \mathrm{~K}$. Subsequently, evaporated molecules are ionized using electron impact ionization and transported into the ion trap via ion gate and quadrupole mass filter into the ion trap. Another oven (neutral molecules source, $\sim 300 \mathrm{~K}$ ) is located under the trap that the vapor phase molecules (anthracene power, J\&K, with a purity better than $99 \%$ ) effuse continuously toward the center of the trap. In the trap, DC/anthracene cluster cations are formed by a collision reaction between cations and neutral anthracene molecule. Helium gas is introduced continuously into 


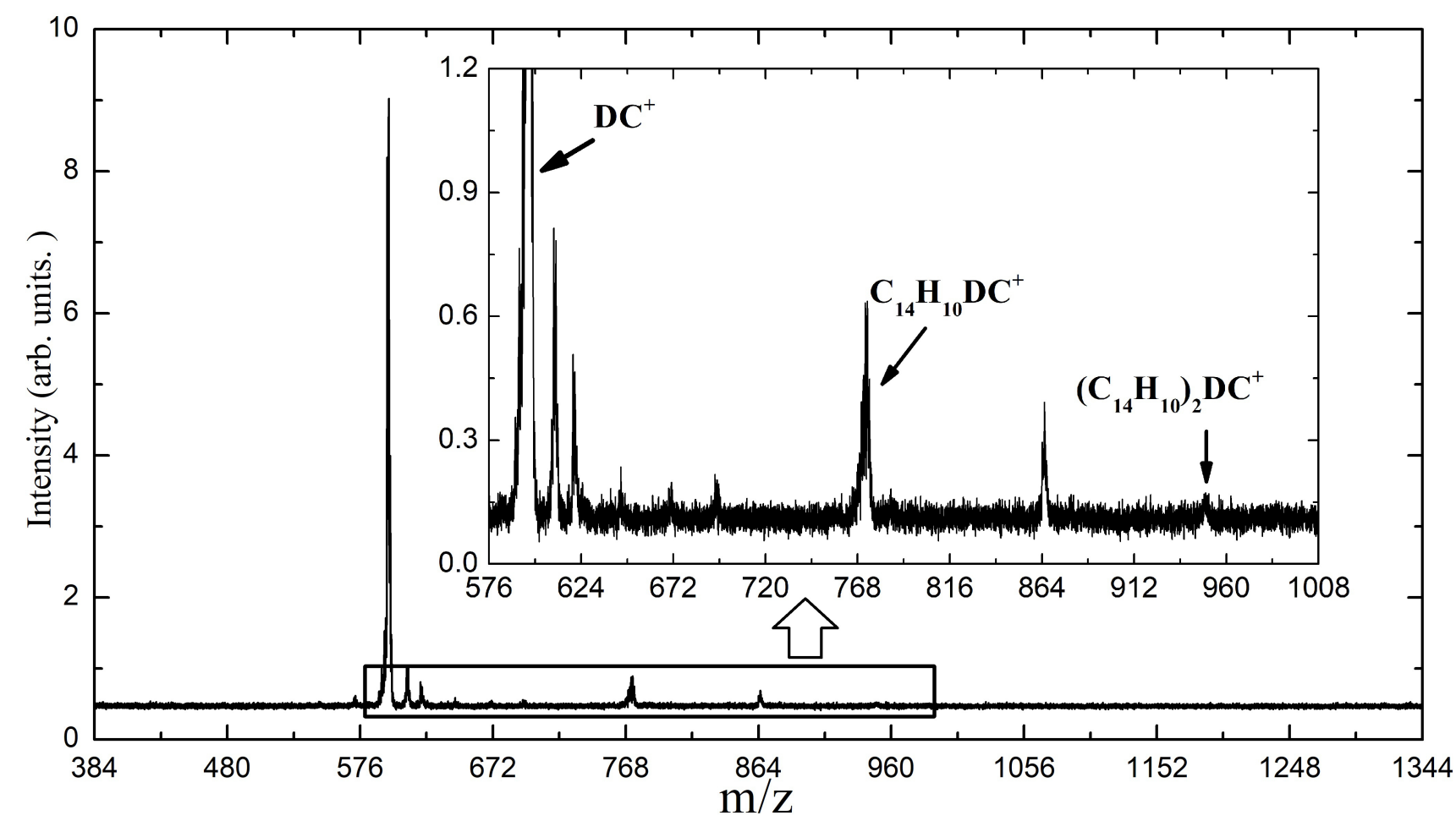

Fig. 1. Mass spectrum of DC/anthracene cluster cations trapped in QIT without laser irradiation. The inset is a zoom-in mass spectrum, revealing the presence of newly formed clusters: $\mathrm{C}_{14} \mathrm{H}_{10} \mathrm{DC}^{+}$and $\left(\mathrm{C}_{14} \mathrm{H}_{10}\right)_{2} \mathrm{DC}^{+}$.

the trap to thermalize the ion cloud through collisions via a leak valve.

The third harmonic of Nd:YAG laser (INDI, SpectraPhysics), $355 \mathrm{~nm}, \sim 6 \mathrm{~ns}$, operated at $10 \mathrm{~Hz}$, is used to irradiate the trapped, newly formed ion clusters. A beam shutter (Uniblitz, XRS-4) acts as a physical shield inside in the chamber and determines the interaction time of the light with the trapped ionic clusters. The shutter is externally triggered to guarantee that the ion cloud is irradiated only for a specified amount of time during each cycle. A high precision delay generator (SRS DG535) controls the full timing sequence.

Our setup operates at a typical frequency of $0.2 \mathrm{~Hz}$, meaning that one full measuring cycle lasts $5 \mathrm{~s}$. We used two time strategies here:

(1) At the leading edge of the master trigger the ion gate opens (0-4.0 s), allowing the ion trap to fill for a certain amount of ions. In this period the trapped ion collision reaction with anthracene molecules to form new cluster cations. Then the beam shutter opens and the ion cloud is irradiated (4.0-4.8 s). At the end of the irradiation, a negative square pulse $(4.88 \mathrm{~s})$ is applied to the end cap of the ion trap, accelerating the ions out of the trap and into the field-free TOF region where the resulting mass fragments are measured. The resulting mass spectrum is shown in Figs. 1 and 2.

(2) At the leading edge of the master trigger the ion gate and beam shutter opens $(0-4.0 \mathrm{~s})$, allowing the ion trap to fill for a certain amount of ions and irradiated by the laser at the same time. Then with a time delay period (4.0-4.8 s), the trapped new photo-formed ions collision reaction with anthracene molecules to form new cluster cations, then the resulting mass is measured (4.88 s). The resulting mass spectrum is shown in Fig. 3.

\section{Experimental results and discussion}

A typical mass spectrum of DC/anthracene cluster cations without laser irradiation is shown in Fig. 1. A series of DC/ anthracene cluster cations are produced, similarly to what was observed for $\mathrm{HBC} /$ anthracene (hexa-peri-hexabenzocoronene, $\left(\mathrm{C}_{42} \mathrm{H}_{18}, \mathrm{HBC}\right)$ in Zhen et al. (2018c). The DC/anthracene cluster cations are labeled in detail in a zoom-in mass spectrum in Fig. 1. The inset spectrum provides more details on the new formed DC/anthracene cluster cations: all of them are dehydrogenated, in other words, partially $\mathrm{H}$ stripped ion species are the most abundant species, that as $\mathrm{C}_{14} \mathrm{H}_{10} \mathrm{DC}^{+}$(e.g., $\mathrm{C}_{14} \mathrm{H}_{10} \mathrm{C}_{48} \mathrm{H}_{19}^{+}, m / z=773$ ) and $\left(\mathrm{C}_{14} \mathrm{H}_{10}\right)_{2} \mathrm{DC}^{+}$ (e.g., $\left.\left(\mathrm{C}_{14} \mathrm{H}_{10}\right)_{2} \mathrm{C}_{48} \mathrm{H}_{18}^{+}, m / z=950\right)$.

Based on the results in Fig. 1 and our previous work in Zhen et al. (2018c), we propose DC/anthracene cluster cations are formed through collision formation reaction between DC fragmented cations and neutral anthracene molecular, and occurs step by step. As for $\mathrm{C}_{14} \mathrm{H}_{10} \mathrm{DC}^{+}$and $\left(\mathrm{C}_{14} \mathrm{H}_{10}\right)_{2} \mathrm{DC}^{+}$, the formation reaction pathways are shown below.

$$
\begin{aligned}
& \mathrm{C}_{14} \mathrm{H}_{10}+\mathrm{DC}^{+} \longrightarrow \mathrm{C}_{14} \mathrm{H}_{10} \mathrm{DC}^{+} \\
& \mathrm{C}_{14} \mathrm{H}_{10}+\mathrm{C}_{14} \mathrm{H}_{10} \mathrm{DC}^{+} \longrightarrow\left(\mathrm{C}_{14} \mathrm{H}_{10}\right)_{2} \mathrm{DC}^{+} .
\end{aligned}
$$

Figure 2 shows the resulting mass spectrum of trapped DC/anthracene cluster cations upon $355 \mathrm{~nm}$ irradiation at $4.0 \mathrm{~mJ}$ laser energy (irradiation times amounting to $0.8 \mathrm{~s}$; i.e., typically approximately eight pulses, from 4.0 to $4.8 \mathrm{~s}$ ). Upon laser irradiation, many new peaks are observed in the mass spectrum, in general, two similar fragmentation sequences can be distinguished in the range of $m / z=384-620$ and $m / z=620$ 800 , respectively. The fragmentation sequence similar to that observed of large PAHs (Zhen et al. 2014).

In the range of $m / z=384-620$, the photo-dissociation of $\mathrm{DC}^{+}$mainly follows dehydrogenation, leading to the species of $\mathrm{C}_{48} \mathrm{H}_{n}^{+}$with $n=[0-19]$. Masses corresponding to $\mathrm{C}_{48-2 * m}^{+}$with $m=[0-7]$ appear as well, all ions are almost completely dehydrogenated, resulting in mass spectrum dominated by species (graphene) with masses by $\mathrm{C}_{2}$ difference (separated by $24 \mathrm{Da}$ mass differences). In the $m / z=620-800$ range, similar to $\mathrm{DC}^{+}$, 


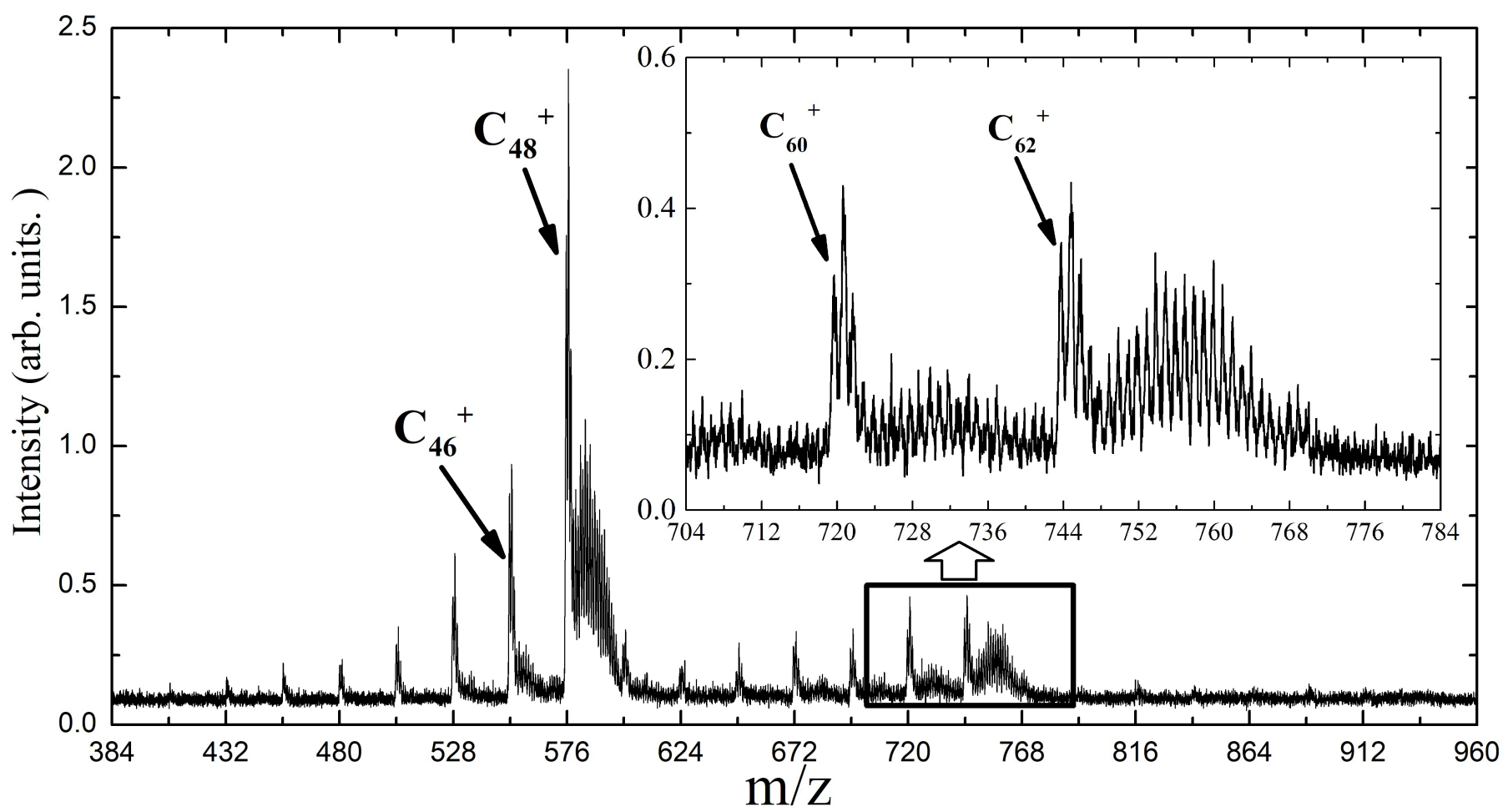

Fig. 2. Mass spectrum of DC/anthracene cluster cations trapped in QIT with laser irradiation upon $355 \mathrm{~nm}$ irradiation at $4.0 \mathrm{~mJ}$ laser energy (irradiation times amounting to $0.8 \mathrm{~s}$, from 4.0 to $4.8 \mathrm{~s}$ ). The inset is a zoom-in mass spectrum is in the range of $m / z=704-784$, revealing the presence of newly formed clusters: $\mathrm{C}_{60 / 62}^{+}$and these dehydrogenated $\mathrm{C}_{14} \mathrm{H}_{10} \mathrm{DC}^{+}$.

the photo-dissociation of $\mathrm{C}_{14} \mathrm{H}_{10} \mathrm{DC}^{+}$mainly follows dehydrogenation, leading to the species of $\mathrm{C}_{60} \mathrm{H}_{n}^{+}$with $n=[0-23]$ and $\mathrm{C}_{62} \mathrm{H}_{m}^{+}$with $m=[0-25]$, as shown in a zoom-in mass spectrum in Fig. 2 in the range of $m / z=704-784$. Masses corresponding to $\mathrm{C}_{62-2 * m}^{+}$with $m=[0-5]$ also appear, resulting in mass spectrum dominated by species (graphene) with masses following a $\mathrm{C}_{2}$ difference. The similar photo-behavior of $\mathrm{C}_{14} \mathrm{H}_{10} \mathrm{DC}^{+}$ to $\mathrm{DC}^{+}$means that the photo-evolution of $\mathrm{C}_{14} \mathrm{H}_{10} \mathrm{DC}^{+}$mainly converts to more aromatic species by dehydrogenation process (e.g., PAHs, such as $\mathrm{C}_{62} \mathrm{H}_{26}$ ) and then to other aromatic species (e.g., graphene, such as $\mathrm{C}_{62}$ and $\mathrm{C}_{60}$ ) (Kroto et al. 1985; Ekern et al. 1998; Castellanos et al. 2018; West et al. 2018; Zhen et al. 2018a).

Based on the above discussion, we propose the photodissociation channel upon laser irradiation for DC cations and $\mathrm{C}_{14} \mathrm{H}_{10} \mathrm{DC}$ cluster cations are shown as below:

$\mathrm{C}_{48} \mathrm{H}_{20}^{+}+\mathrm{h} v \longrightarrow \mathrm{C}_{48} \mathrm{C}_{n}^{+}, n=[0-19]$

$\mathrm{C}_{14} \mathrm{H}_{10} \mathrm{DC}^{+}+\mathrm{h} v \longrightarrow \mathrm{C}_{62} \mathrm{H}_{26}^{+}$

$\mathrm{C}_{62} \mathrm{H}_{26}^{+}+\mathrm{h} v \longrightarrow \mathrm{C}_{62} \mathrm{H}_{m}^{+}, m=[0-25]$.

Figure 3 shows the resulting mass spectrum of trapped DC/anthracene cluster cations upon $355 \mathrm{~nm}$ irradiation at $4.0 \mathrm{~mJ}$ laser energy (irradiation times amounting to $4.0 \mathrm{~s}$; i.e., typically $\sim 40$ pulses, from 0 to $4.0 \mathrm{~s}$ ) and then collision reaction with neutral anthracene molecular (collision reaction times amounting to $0.8 \mathrm{~s}$, from 4.0 to $4.8 \mathrm{~s}$ ). Upon laser irradiation and then with the collision reaction, a series of cationic clusters are generated and many more new peaks (three groups) are observed in the mass spectrum in the range of $m / z=620-1344$ (Fig. 3, upper panel A).

Further detail on the three groups of new formation species are labeled and illustrated in three zoom-in mass spectra in Figs. 3B-D, respectively. In Fig. 3B, $\mathrm{C}_{14} \mathrm{H}_{10} \mathrm{C}_{46}^{+}$ $(m / z=730)$ and $\mathrm{C}_{14} \mathrm{H}_{10} \mathrm{C}_{48} \mathrm{H}_{(0-19)}^{+}(m / z=754-773)$ are produced, and formed through the fragmented cations $\left(\mathrm{C}_{46}^{+}\right.$and $\left.\mathrm{C}_{48} \mathrm{H}_{(0-19)}^{+}\right)$, that are generated from the fragmentation process of $\mathrm{DC}^{+}$, collision reaction with neutral anthracene molecular. $\mathrm{C}_{60}^{+}(m / z=720)$ and $\mathrm{C}_{62}^{+}(\mathrm{m} / z=744)$ are produced, and formed through the photo-dissociation of $\mathrm{C}_{14} \mathrm{H}_{10} \mathrm{DC}^{+}$. In Fig. 3C, $\left(\mathrm{C}_{14} \mathrm{H}_{10}\right)_{2} \mathrm{C}_{46}^{+}(\mathrm{m} / \mathrm{z}=908), \mathrm{C}_{14} \mathrm{H}_{10} \mathrm{C}_{62}^{+} \quad(\mathrm{m} / \mathrm{z}=922)$ and $\left(\mathrm{C}_{14} \mathrm{H}_{10}\right)_{2} \mathrm{C}_{48}^{+}(\mathrm{m} / z=932)$ are produced, and formed by $\mathrm{C}_{14} \mathrm{H}_{10} \mathrm{C}_{46}^{+}, \mathrm{C}_{62}^{+}$and $\mathrm{C}_{14} \mathrm{H}_{10} \mathrm{C}_{48}^{+}$collision reaction with neutral anthracene molecular. $\mathrm{C}_{76}^{+}(\mathrm{m} / \mathrm{z}=912)$ is produced, and formed by the photo-dissociation of $\mathrm{C}_{14} \mathrm{H}_{10} \mathrm{C}_{62}^{+}$. In Fig. 3D, $\left(\mathrm{C}_{14} \mathrm{H}_{10}\right)_{3} \mathrm{C}_{46}^{+} \quad(\mathrm{m} / \mathrm{z}=1086), \mathrm{C}_{14} \mathrm{H}_{10} \mathrm{C}_{76}^{+} \quad(\mathrm{m} / \mathrm{z}=1090)$, $\left(\mathrm{C}_{14} \mathrm{H}_{10}\right)_{2} \mathrm{C}_{62}^{+}(\mathrm{m} / \mathrm{z}=1100)$ and $\left(\mathrm{C}_{14} \mathrm{H}_{10}\right)_{3} \mathrm{C}_{48}^{+}(\mathrm{m} / \mathrm{z}=1110)$ are produced, and formed by $\left(\mathrm{C}_{14} \mathrm{H}_{10}\right)_{2} \mathrm{C}_{46}^{+}, \mathrm{C}_{76}^{+}, \mathrm{C}_{14} \mathrm{H}_{10} \mathrm{C}_{48}^{+}$ and $\left(\mathrm{C}_{14} \mathrm{H}_{10}\right)_{2} \mathrm{C}_{48}^{+}$collision reaction with neutral anthracene molecular.

Based on the discussion above, we propose the formation and photo-dissociation under laser irradiation for these new species in different time range are shown as below:

In the time range from 0 to $4.0 \mathrm{~s}$, the photo-dissociation and formation behavior is similar to what we see in Fig. 2 (we may have a lower intensify of formed DC/anthracene cluster cations), we summarize the formation reaction pathway for some new species are shown as below:

$\mathrm{C}_{48} \mathrm{H}_{20}^{+}+\mathrm{h} v \rightarrow \mathrm{C}_{48} \mathrm{H}_{n}^{+}, n=[0-19]$

$\mathrm{C}_{14} \mathrm{H}_{10} \mathrm{DC}^{+}+\mathrm{h} v \longrightarrow \mathrm{C}_{62} \mathrm{H}_{26}^{+}$

$\mathrm{C}_{62} \mathrm{H}_{26}^{+}+\mathrm{h} v \longrightarrow \mathrm{C}_{62} \mathrm{H}_{m}^{+}, m=[0-25]$.

In the time range from 4.0 to $4.8 \mathrm{~s}$, we propose that new cluster cations are formed through a collision formation reaction between fragmented cations (shown as above) and neutral 


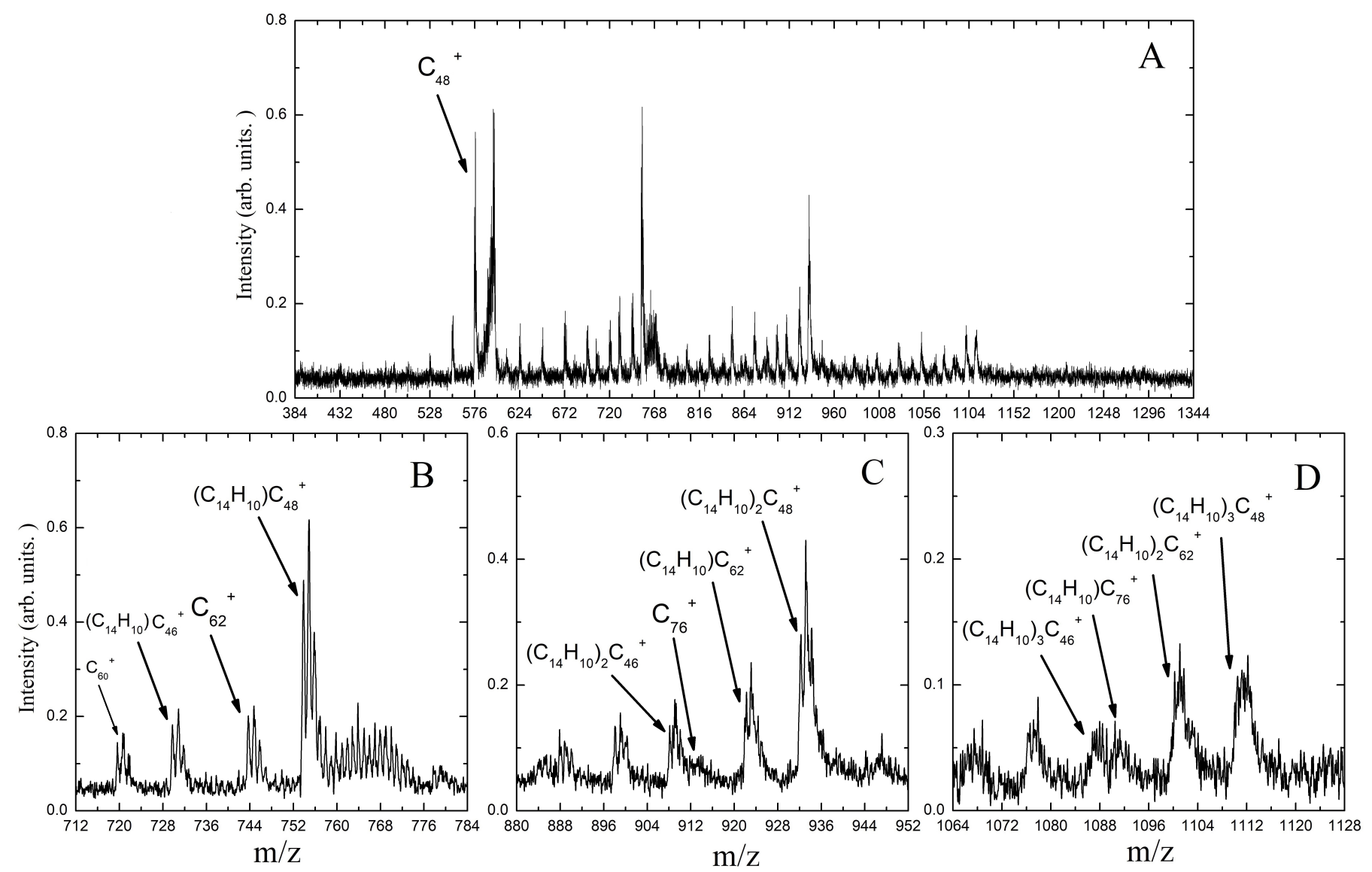

Fig. 3. Mass spectrum of DC/anthracene cluster cations trapped in QIT with laser irradiation upon $355 \mathrm{~nm}$ irradiation at $4.0 \mathrm{~mJ}$ laser energy (irradiation times amounting to $4.0 \mathrm{~s}$, from 0 to $4.0 \mathrm{~s}$ ), and then collision reaction in the period of 4.0-4.8 s. Panel A: whole mass spectrum in the range of $m / z=384-1344$. Panels $B, C$, and $D$ : zoom-ins of the mass spectrum, revealing the details of the presence newly formed clusters.

anthracene molecular, we summarize the formation reaction pathway for some new species below.

$\mathrm{C}_{14} \mathrm{H}_{10}+\mathrm{C}_{48} \mathrm{H}_{n}^{+} \longrightarrow\left(\mathrm{C}_{14} \mathrm{H}_{10}\right) C_{48} \mathrm{H}_{n}^{+}, n=[0-19]$

$\mathrm{C}_{14} \mathrm{H}_{10}+\mathrm{C}_{62} \mathrm{H}_{m}^{+} \longrightarrow\left(\mathrm{C}_{14} \mathrm{H}_{10}\right) \mathrm{C}_{62} \mathrm{H}_{m}^{+}, m=[0-25]$.

\section{Astronomical implications and conclusions}

The origin and evolution of large molecular (e.g., PAH clusters or graphene) in space, especially in the range of $\sim 100$ atoms or nanometer-size, is a very interesting topic. It has been suggested that the presence of large molecular may help explain certain characteristics of the astronomical observations of the IR spectra (Tielens 2013). While the formation process of such clusters in the experiment is not entirely clear and hence it is difficult to assess their formation in the ISM (Candian et al. 2018). Different from the energetic ions induce intra-cluster reactions to form large molecules, in other words, ion-driven (Zettergren et al. 2013; Delaunay et al. 2015). Our experimental results presented here provide further insight on the formation process of large molecular in the ISM. New larger molecules can be built up under the promotion of irradiation by laser, in other words, photo-driven, the conversion is suggested to take place in the areas of high luminosity where PAH clusters absorb multiple photons. The multiphoton absorption leads to the dehydrogenation of PAHs with the following polymerization with smaller PAHs. The DC/anthracene clusters, as an example of initial precursor molecular, the formation mechanism (gas-phase condensation) and photo-dissociation behavior obtained in here can be generalized and provides a reasonable explanation for the formation and evolution of large molecular in space.

We stress that given the new species produced in this work, PAH cluster cations (e.g., $\left(\mathrm{C}_{14} \mathrm{H}_{10}\right) \mathrm{C}_{48} \mathrm{H}_{n}^{+}, n=[1-19]$ and $\left.\left(\mathrm{C}_{14} \mathrm{H}_{10}\right) \mathrm{C}_{62} \mathrm{H}_{m}^{+}, m=[1-25]\right)$ and $\mathrm{PAH}$-graphene cluster cations (e.g., $\left(\mathrm{C}_{14} \mathrm{H}_{10}\right)_{n} \mathrm{C}_{48}^{+}, n=0,1,2,3$ and $\left.\left(\mathrm{C}_{14} \mathrm{H}_{10}\right)_{m} \mathrm{C}_{62}^{+}, m=0,1,2\right)$, range from 48 to 120 atoms or $\sim 2 \mathrm{~nm}$ in size, that can be a nice example of carbonaceous dust grains in the ISM (Kroto et al. 1985; Henning \& Salama 1998; Jäger et al. 2009, 2011). The newly formed species produced here offer an approach of carbonaceous dust grains that provide a basic understanding (e.g., composition and structural properties) of the grain formation processes in the gas-phase.

Finally, in conclusion, we have presented the experimental results on the large interstellar molecules (e.g., a family group of molecular clusters and graphene). With the photodissociation by the laser and with the formation pathway by gas-phase condensation (collision reaction), PAH clusters are evolving to become more aromatic, structurally complicated, and larger. Theoretical studies regarding the dynamic pathway will be carried out in a subsequent paper (Zhang \& Zhen, in prep.).

Acknowledgements. This work is supported by the Fundamental Research Funds for the Central Universities and from the National Science Foundation of China (NSFC, Grant No. 11421303 and Grant No. 11590782). 


\section{References}

Allamandola, L. J., Tielens, A. G. G. M., \& Barker, J. R. 1989, ApJS, 71, 733 Berné, O., \& Tielens, A. G. G. M. 2012, Proc. Natl. Acad. Sci., 109, 401

Berné, O., Joblin, C., Deville, Y., et al. 2007, A\&A, 469, 575

Candian, A., Zhen, J., \& Tielens, A. G. G. M. 2018, Phys. Today, 71, 38

Castellanos, P., Candian, A., Zhen, J., Linnartz, H., \& Tielens, A. G. G. M. 2018, A\&A, 616, A166

Chen, T., Gatchell, M., Stockett, M. H., et al. 2015, J. Chem. Phys., 142, 144305

Croiset, B. A., Candian, A., Berné, O., \& Tielens, A. G. G. M. 2016, A\&A, 590, A26

Delaunay, R., Gatchell, M., Rousseau, P., et al. 2015, J. Phys. Chem. Lett., 6 , 1536

Ekern, S. P., Marshall, A. G., Szczepanski, J., \& Vala, M. 1998, J. Phys. Chem. A, 102,3498

Henning, T., \& Salama, F. 1998, Science, 282, 2204

Hollenbach, D. J., \& Tielens, A.G.G.M. 1999, Rev. Mod. Phys., 71, 173

Jäger, C., Huisken, F., Mutschke, H., Llamas Jansa, I., \& Henning, T. 2009, ApJ, 696, 706

Jäger, C., Mutschke, H., Henning, T., \& Huisken, F. 2011, EAS Pub. Ser., 46, 293

Kroto, H., Heath, J., O’Brien, S., Curl, R., \& Smalley, R. 1985, Nature, 318, 162

Malloci, G., Mulas, G., Cecchi-Pestellini, C., \& Joblin, C. 2008, A\&A, 489, 1183
Micelotta, E. R., Jones, A. P., \& Tielens, A. G. G. M. 2010, A\&A, 510, A37

Pety, J., Teyssier, D., Fossé, D., et al. 2005, A\&A, 435, 885

Pilleri, P., Joblin, C., Boulanger, F., \& Onaka, T. 2015, A\&A, 577, A16

Puget, J. L., \& Leger, A. 1989, ARA\&A, 27, 161

Rapacioli, M., Joblin, C., \& Boissel, P. 2005, A\&A, 429, 193

Rapacioli, M., Calvo, F., Joblin, C., et al. 2006, A\&A, 460, 519

Ricca, A., Bauschlicher, C. W., Jr., Boersma, C., Tielens, A. G. G. M., \& Allamandola, L. J. 2012, ApJ, 754, 75

Richter, H., Granata, S., Green, W.H., \& Howard, J.B. 2004, PCI, 30, 1397

Rhee, Y. M., Lee, T. J., Gudipati, M. S., Allamandola, L. J. \& Head-Gordon, M. 2007, Proc. Natl. Acad. Sci., 104, 5274

Sellgren, K. 1984, ApJ, 277, 623

Tielens, A. G. G. M. 2008, ARA\&A, 46, 289

Tielens, A. G. G .M. 2013, Rev. Mod. Phys., 85, 1021

van Diedenhoven, B., Peeters, E., Van Kerckhoven, C., et al. 2004, ApJ, 611, 928

West, B., Castillo, S. R., Sit, A., et al. 2018, Phys. Chem. Chem. Phys., 20, 7195

Zettergren, H, Johansson, H. A. B., Schmidt, H. T., et al. 2010, J. Chem. Phys., 133, 104301

Zettergren, H., Rousseau, P., Wang, Y., et al. 2013, Phys. Rev. Lett., 110, 185501

Zhen, J., Paardekooper, D. M., Candian, A., Linnartz, H., \& Tielens, A. G. G. M. 2014, Chem. Phys. Lett., 592, 211

Zhen, J., Chen, T., \& Tielens, A. G. G. M. 2018a, ApJ, 863, 128

Zhen, J., Candian, A., Castellanos, P., et al. 2018b, ApJ, 854, 27

Zhen, J., Zhang, W. W., \& Yang, Y. Y. 2018c, ApJ, submitted 